\title{
Sparse partial robust M regression
}

\author{
Irene Hoffmann ${ }^{\mathrm{a}, *}$, Sven Serneels ${ }^{\mathrm{b}}$, Peter Filzmoser ${ }^{\mathrm{a}}$, Christophe Croux ${ }^{\mathrm{c}}$ \\ ${ }^{a}$ Institute of Statistics and Mathematical Methods in Economics, TU Wien, Vienna \\ 1040, Austria \\ ${ }^{b}$ BASF Corp., Tarrytown, NY 10591, USA \\ ${ }^{c}$ Faculty of Economics and Business, KU Leuven, B3000 Leuven, Belgium
}

\begin{abstract}
Sparse partial robust M regression is introduced as a new regression method. It is the first dimension reduction and regression algorithm that yields estimates with a partial least squares like interpretability that are sparse and robust with respect to both vertical outliers and leverage points. A simulation study underpins these claims. Real data examples illustrate the validity of the approach.
\end{abstract}

Keywords: Biplot, Partial least squares, Robustness, Sparse estimation

\section{Introduction}

Sparse regression methods have been a major topic of research in statistics over the last decade. They estimate a linear relationship between a predictand $\boldsymbol{y} \in \mathbb{R}^{n}$ and a predictor data matrix $\boldsymbol{X} \in \mathbb{R}^{n \times p}$. Assuming the linear model

$$
\boldsymbol{y}=\boldsymbol{X} \boldsymbol{\beta}+\varepsilon,
$$

the classical estimator is given by solving the least squares criterion

$$
\hat{\boldsymbol{\beta}}=\underset{\boldsymbol{\beta}}{\operatorname{argmin}}\|\boldsymbol{y}-\boldsymbol{X} \boldsymbol{\beta}\|^{2}
$$

${ }^{*}$ Correspondence to I. Hoffmann, TU Wien, Wiedner Hauptstraße 8-10/105, A-1040 Wien, Austria.

Email addresses: irene.hoffmann@tuwien.ac.at (Irene Hoffmann), sven.serneels@basf.com (Sven Serneels), p.filzmoser@tuwien.ac.at (Peter Filzmoser), christophe.croux@econ.kuleuven. be (Christophe Croux) 
with the squared $L_{2}$ norm $\|\boldsymbol{u}\|^{2}=\sum_{i=1}^{p} u_{i}^{2}$ for any vector $\boldsymbol{u} \in \mathbb{R}^{p}$. Thereby the predicted responses are $\hat{\boldsymbol{y}}=\boldsymbol{X} \hat{\boldsymbol{\beta}}$. When the predictor data contain a column of ones, the model incorporates an intercept.

Typically, but not exclusively, when $p$ is large, the $\boldsymbol{X}$ data matrix tends to contain columns of uninformative variables, i.e. variables that bear no information related to the predictand. Estimates of $\boldsymbol{\beta}$ often have a subset of components $\left\{\hat{\beta}_{j_{1}}, \ldots, \hat{\beta}_{j_{\check{p}}}\right\}$ of small magnitude corresponding to $\check{p}$ uninformative variables. As these components are small but not exactly zero, each of them still contributes to the model and, more importantly, to increased estimation and prediction uncertainty. In contrast, a sparse estimator of $\boldsymbol{\beta}$ will have many components that are exactly equal to zero.

Penalized regression methods impose conditions on the norm of the coefficient vector. The Lasso estimate [1], where an $L_{1}$ penalty term is used, leads to a sparse coefficient vector:

$$
\min _{\boldsymbol{\beta}}\|\boldsymbol{y}-\boldsymbol{X} \boldsymbol{\beta}\|^{2}+\lambda_{1}\|\boldsymbol{\beta}\|_{1}
$$

with $\|\boldsymbol{u}\|_{1}=\sum_{i=1}^{p}\left|u_{i}\right|$ for any vector $\boldsymbol{u} \in \mathbb{R}^{p}$. The nonnegative tuning parameter $\lambda_{1}$ determines the sparsity of the estimation and implicitly reflects the size of $\check{p}$. The Lasso sparse regression estimate has become a statistical regression tool of widespread application, especially in fields of research where data dimensionality is typically high, such as chemometrics, cheminformatics or bioinformatics [2]. But, since it is nonrobust, it may be severely distorted by outliers in the data.

Robust multiple regression has attracted widespread attention from statisticians since as early as the 1970s. For an overview of robust regression methods, we refer to e.g. Maronna et al. [3]. However, only recently, robust sparse regression estimators have been proposed. One of the few existing sparse and robust regression estimators that is robust to both vertical outliers (outliers in the predictand) and leverage points (outliers in the predictor data), is sparse least trimmed squares regression [4], which is a sparse penalized version of the least trimmed squares (LTS) robust regression estimator $[5]$.

In applied sciences, there is often a need for both regression analysis, and interpretative analysis. In order to visualize the data and to interpret the high-dimensional structure(s) in them, it is customary to project the predictor data onto a limited set of latent components and then analyze the 
individual cases' position as well as how each original variable contributes to the latent components in a biplot. A first approach would be to do a (potentially sparse) principal component analysis, followed by a (potentially sparse) regression. The main issue with that approach is that the principal components are defined according to a maximization criterion that does not account for the predictand. With this reason, partial least squares regression (PLS) [6] has become a mainstay tool in applied sciences such as chemometrics. It provides a projection onto a few latent components that can be visualized in biplots, and it yields a vector of regression coefficients based on those latent components.

Partial least squares regression is both a nonrobust and a nonsparse estimator. Manifold proposals to robustify PLS have been discussed, of which a good overview is given in Filzmoser et al. [7]. One of the most widely applied robust alternatives to PLS is partial robust M regression [8]. Likely its popularity is due to the fact that it provides a fair tradeoff between statistical robustness with respect to both vertical outliers and leverage points on the one hand and statistical and computational efficiency on the other hand. From an application perspective, it has been reported to perform well [9]. Introduction of sparseness into the partial least squares framework is a more recent topic of research $[10,11,12]$.

In this article, a novel estimator is introduced, called Sparse Partial Robust $M$ regression, which is up to our knowledge the first estimator to offer all three benefits simultaneously: (i) it is based on projection onto latent structures and thereby yields PLS like visualization, (ii) it is integrally sparse, yielding not only regression coefficients with exact zero components, but also sparse direction vectors, and (iii) it is robust with respect to both vertical outliers and leverage points.

\section{The sparse partial robust $M$ regression estimator}

The sparse partial robust M regression (SPRM) estimator can be viewed at as either a sparse version of the partial robust $M$ regression (PRM) estimator [8], or as a way to robustify the sparse PLS (SPLS) estimator [11]. Therefore, its construction inherits some characteristics from both precursors.

In partial least squares, the latent components (or scores) $\boldsymbol{T}$ are defined as linear combinations of the original variables $\boldsymbol{T}=\boldsymbol{X} \boldsymbol{A}$, wherein the so-called direction vectors $\boldsymbol{a}_{h}$ (in the PLS literature also known as weighting vectors) 
are the columns of $\boldsymbol{A}$. The direction vectors maximize squared covariance to the predictand:

$$
\boldsymbol{a}_{h}=\underset{\boldsymbol{a}}{\operatorname{argmax}} \operatorname{cov}^{2}(\boldsymbol{X} \boldsymbol{a}, \boldsymbol{y}),
$$

for $h \in\left\{1, \ldots, h_{\max }\right\}$ under the constraints that

$$
\left\|\boldsymbol{a}_{h}\right\|=1 \text { and } \boldsymbol{a}_{h}^{T} \boldsymbol{X}^{T} \boldsymbol{X} \boldsymbol{a}_{i}=0 \text { for } 1 \leq i<h .
$$

Here, $h_{\max }$ is the maximum number of components we want to retrieve. We assume throughout the article, that both predictor and predictand variables are centered, so that

$$
\operatorname{cov}^{2}(\boldsymbol{X} \boldsymbol{a}, \boldsymbol{y})=\frac{1}{(n-1)^{2}} \boldsymbol{a}^{T} \boldsymbol{X}^{T} \boldsymbol{y} \boldsymbol{y}^{T} \boldsymbol{X} \boldsymbol{a}=\frac{1}{(n-1)^{2}} \boldsymbol{a}^{T} \boldsymbol{M}^{T} \boldsymbol{M} \boldsymbol{a}
$$

with $\boldsymbol{M}=\boldsymbol{y}^{T} \boldsymbol{X}$. Regressing the dependent variable onto the scores, yields

$$
\hat{\gamma}=\underset{\gamma}{\operatorname{argmin}}\|\boldsymbol{y}-\boldsymbol{T} \boldsymbol{\gamma}\|^{2}=\left(\boldsymbol{T}^{T} \boldsymbol{T}\right)^{-1} \boldsymbol{T}^{T} \boldsymbol{y}
$$

Then, since $\hat{\boldsymbol{y}}=\boldsymbol{T} \hat{\boldsymbol{\gamma}}$ and $\boldsymbol{T}=\boldsymbol{X} \boldsymbol{A}$, one gets $\hat{\boldsymbol{\beta}}=\boldsymbol{A} \hat{\boldsymbol{\gamma}}$.

In order to obtain a robust version of the partial least squares estimator, case weights $\omega_{i}$ are assigned to the rows of $\boldsymbol{X}$ and $\boldsymbol{y}$. Let

$$
\tilde{\boldsymbol{X}}=\boldsymbol{\Omega} \boldsymbol{X} \quad \text { and } \quad \tilde{\boldsymbol{y}}=\boldsymbol{\Omega} \boldsymbol{y}
$$

with $\Omega$ a diagonal matrix with diagonal elements $\omega_{i} \in[0,1]$ for $i \in\{1, \ldots, n\}$. Outlying observations will receive a weight lower than one. An observation is an outlier when it has a large residual, or a large value of the covariate (hence a large leverage) in the latent regression model (i.e. the regression of the predictand on the latent components). Let $\boldsymbol{t}_{i}$ denote the rows of $\boldsymbol{T}$, $r_{i}=y_{i}-\boldsymbol{t}_{i}^{T} \hat{\gamma}$ are the residuals of the latent variable regression model, where $y_{i}$ are the elements of the vector $\boldsymbol{y}$. Let $\hat{\sigma}$ denote a robust scale estimator of the residuals; we take the median absolute deviation (MAD). Then the weights are defined by

$$
\omega_{i}^{2}=\omega_{R}\left(\frac{r_{i}}{\hat{\sigma}}\right) \omega_{T}\left(\frac{\left\|\boldsymbol{t}_{i}-\operatorname{med}_{j}\left(\boldsymbol{t}_{\boldsymbol{j}}\right)\right\|}{\operatorname{med}_{i}\left\|\boldsymbol{t}_{i}-\operatorname{med}_{j}\left(\boldsymbol{t}_{\boldsymbol{j}}\right)\right\|}\right) .
$$

More specifics on weight functions $\omega_{R}$ and $\omega_{T}$ will be discussed in Section 3. 
With (5) and $\tilde{\boldsymbol{M}}=\tilde{\boldsymbol{y}}^{T} \tilde{\boldsymbol{X}}$, the robust maximization criterion for the direction vectors is

$$
\boldsymbol{a}_{h}=\underset{\boldsymbol{a}}{\operatorname{argmax}} \boldsymbol{a}^{T} \tilde{\boldsymbol{M}}^{T} \tilde{\boldsymbol{M}} \boldsymbol{a},
$$

under the constraints that

$$
\left\|\boldsymbol{a}_{h}\right\|=1 \text { and } \boldsymbol{a}_{h}^{T} \tilde{\boldsymbol{X}}^{T} \tilde{\boldsymbol{X}} \boldsymbol{a}_{i}=0 \text { for } 1 \leq i<h,
$$

which is identical to maximization criterion (4) if $\Omega$ is the identity matrix.

In order to obtain a fully robust PLS estimation, the latent variable regression needs to be robustified too. Thereunto, note that the ordinary least squares minimization criterion can be written as

$$
\hat{\boldsymbol{\gamma}}=\underset{\gamma}{\operatorname{argmin}} \sum_{i=1}^{n} \rho\left(y_{i}-\boldsymbol{t}_{i}^{T} \boldsymbol{\gamma}\right),
$$

with $\rho(u)=u^{2}$. Using a $\rho$ function with bounded derivative in criterion (10) yields a well-known class of robust regression estimators called $M$ estimators. They are computed as iteratively reweighted LS-estimators, with weight function $\omega(u)=\rho^{\prime}(u) / u$. The resulting estimator is the partial robust $\mathrm{M}$ regression estimator [8].

Imposing sparseness on the PRM estimator can now be achieved by setting an $L_{1}$ penalty to the direction vectors $\boldsymbol{a}_{h}$ in (9a). To get sufficiently sparse estimates the sparseness is imposed on a surrogate direction vector $\boldsymbol{c}$ instead [13]. More specifically,

$$
\min _{\boldsymbol{c}, \boldsymbol{a}}-\kappa \boldsymbol{a}^{T} \tilde{\boldsymbol{M}}^{T} \tilde{\boldsymbol{M}} \boldsymbol{a}+(1-\kappa)(\boldsymbol{c}-\boldsymbol{a})^{T} \tilde{\boldsymbol{M}}^{T} \tilde{\boldsymbol{M}}(\boldsymbol{c}-\boldsymbol{a})+\lambda_{1}\|\boldsymbol{c}\|_{1}
$$

under the constraints that

$$
\left\|\boldsymbol{a}_{h}\right\|=1 \text { and } \boldsymbol{a}_{h}^{T} \tilde{\boldsymbol{X}}^{T} \tilde{\boldsymbol{X}} \boldsymbol{a}_{i}=0 \text { for } 1 \leq i<h .
$$

The final estimate of the direction vector is given by

$$
\boldsymbol{a}_{h}=\frac{\hat{\boldsymbol{c}}}{\|\hat{\boldsymbol{c}}\|}
$$

with $\hat{\boldsymbol{c}}$ is the surrogate vector minimizing (11a). In this way, we obtain a sparse matrix of robustly estimated direction vectors $\boldsymbol{A}$ and scores $\boldsymbol{T}=$ 
$\boldsymbol{X} \boldsymbol{A}$. After regressing the dependent variable on the latter using criterion (10) we get the sparse partial robust M regression estimator. Note that the sparsity of the estimated directions carries through to the vector of regression coefficients.

This definition leads to a complex optimization task in which three parameters need to be selected $h_{\max }, \kappa$ and $\lambda_{1}$. Nevertheless, Chun and Keles [11] have shown that the optimization problem does not depend on $\kappa$ for any $\kappa \in(0,1 / 2]$ for univariate $\boldsymbol{y}$ (which is the case throughout this article). Therefore, the three parameter search reduces to the number of latent components $h_{\text {max }}$ and the sparsity parameter $\lambda_{1}$. How these parameters can be selected will be discussed in detail in Section 4 . The next section outlines a fast algorithm to compute the SPRM estimator.

\section{The SPRM algorithm}

The SPRM estimator can be implemented in a surprisingly straightforward manner. Chun and Keleş [11] have shown that imposing sparsity on PLS estimates according to criterion (11) yields analytically exact solutions. Denote by $\boldsymbol{z}_{h}$ the classical, nonsparse PLS direction vectors of the deflated $\boldsymbol{X}$ matrix, i.e. $\boldsymbol{z}_{h}=\boldsymbol{E}_{h}^{T} \boldsymbol{y} /\left\|\boldsymbol{E}_{h}^{T} \boldsymbol{y}\right\|$, wherein $\boldsymbol{E}_{h}$ is $\boldsymbol{X}$ deflated in order to fulfil the orthogonality side constraints in (11b). Hence, $\boldsymbol{E}_{1}=\boldsymbol{X}$ and $\boldsymbol{E}_{h+1}=\boldsymbol{E}_{h}-\boldsymbol{t}^{h} \boldsymbol{t}^{h T} \boldsymbol{E}_{h} /\left\|\boldsymbol{t}^{h}\right\|^{2}$ where $\boldsymbol{t}^{h}$ is the score vector computed in the previous step. Then the exact SPLS solution is given by

$$
\boldsymbol{w}_{h}=\left(\left|\boldsymbol{z}_{h}\right|-\lambda_{1} / 2\right) \odot \boldsymbol{I}\left(\left|\boldsymbol{z}_{h}\right|-\lambda_{1} / 2>0\right) \odot \operatorname{sgn}\left(\boldsymbol{z}_{h}\right),
$$

wherein $\boldsymbol{I}(\cdot)$ denotes the indicator function that yields a vector whose elements equal 1 if the argument is true and 0 otherwise, and $\odot$ denotes the Hadamard (element wise) vector product. In (13), $\left|\boldsymbol{z}_{h}\right|$ is the vector of the absolute values of the components of $\boldsymbol{z}_{h}$, and $\operatorname{sgn}\left(\boldsymbol{z}_{h}\right)$ is the vector of the signs of the components. By putting the vectors $\boldsymbol{w}_{h}$ in the columns of $\boldsymbol{W}$ for $h=1, \ldots, h_{\max }$, the sparse direction vectors in terms of the original, not deflated variables are given by $\boldsymbol{A}=\boldsymbol{W}\left(\boldsymbol{W}^{T} \boldsymbol{X}^{T} \boldsymbol{X} \boldsymbol{W}\right)^{-1}$.

Formula (13) can be replaced by an equivalent expression. Let $\eta$ denote a tuning parameter with $\eta \in[0,1)$. Then we redefine

$$
\boldsymbol{w}_{h}=\left(\left|\boldsymbol{z}_{h}\right|-\eta \max _{i}\left|z_{i h}\right|\right) \odot \boldsymbol{I}\left(\left|\boldsymbol{z}_{h}\right|-\eta \max _{i}\left|z_{i h}\right|>0\right) \odot \operatorname{sgn}\left(\boldsymbol{z}_{h}\right),
$$

with $z_{i h}$ being the components of $\boldsymbol{z}_{h}$. The parameter $\eta$ determines the size of the threshold, as a fraction of the maximum of $\boldsymbol{z}_{h}$, beneath which all elements 


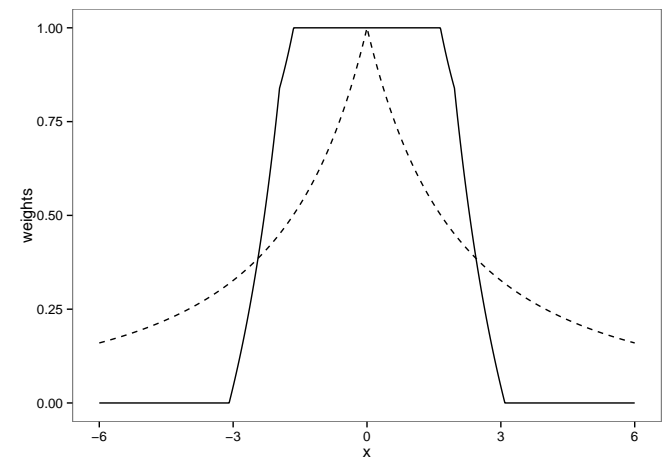

Figure 1: The Hampel (solid) weighting function with standard normal 95\%, 97.5\% and 99.9\% quantiles as cutoffs and the Fair (dashed) weighting function with parameter $c=4$.

of vector $\boldsymbol{w}_{h}$ are set to zero. Since the range of $\eta$ is known in this definition, it facilitates the tuning parameter selection via cross validation (see Section 4).

Computation of the M estimators in (10) boils down to iteratively reweighting the least squares estimator. We use the redescending Hampel weighting function giving a good trade-off between robustness and efficiency [14].

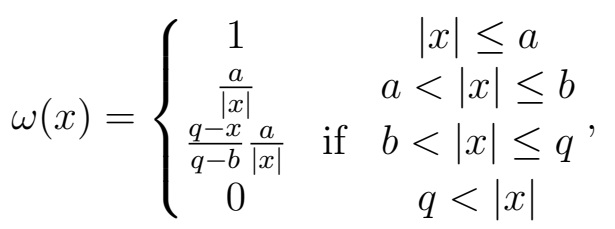

wherein the tuning constants $a, b$ and $q$ can be chosen as distribution quantiles. For the residual weight function $\omega_{R}$ in (8) we take the $0.95,0.975$ and 0.999 quantiles of the standard normal, for $\omega_{T}$ the corresponding quantile of a chi-square distribution.

Note that in the original publication on partial robust $M$ regression [8], the Fair function was recommended (both weighting functions are plotted in Figure 1), but the authors consider the Hampel redescending function superior over the Fair function, because (i) it yields case weights that are much easier to interpret, since they are exactly 1 for the regular cases, exactly 0 for the severe outliers and in the interval $(0,1)$ for the moderate outliers and because (ii) the tuning constants for the cutoffs can be set according to intuitively understandable statistical values such as quantiles from a corresponding distribution function. 
$\boldsymbol{X}$ and $\boldsymbol{y}$ denote robustly centered data (by column-wise median).

1. Calculate initial case weights:

- Calculate distances for $\boldsymbol{x}_{i}(i$ th row of $\boldsymbol{X})$ and $y_{i}$ :

$$
\begin{array}{ll}
d_{i}=\frac{\left\|\boldsymbol{x}_{i}\right\|}{\operatorname{med}_{j}\left\|\boldsymbol{x}_{j}\right\|} & \text { and } \\
r_{i}=\frac{\left|y_{i}\right|}{c \operatorname{med}_{j}\left|y_{j}\right|} \quad \text { for } i \in\{1, \ldots, n\}
\end{array}
$$

where $c=1.4826$ for consistency of the MAD.

- Define initial weights $\omega_{i}=\sqrt{\omega_{T}\left(d_{i}\right) \omega_{R}\left(r_{i}\right)}$ for $\Omega$ (see (8)).

2. Iteratively reweighting:

- Weight data:

$$
\begin{aligned}
& \boldsymbol{X}_{\omega}=\Omega \boldsymbol{X} \\
& \boldsymbol{y}_{\omega}=\Omega \boldsymbol{y}
\end{aligned}
$$

- Apply the sparse NIPALS to $\boldsymbol{X}_{\omega}$ and $\boldsymbol{y}_{\omega}$ and obtain scores $\boldsymbol{T}_{\omega}$, directions $\boldsymbol{A}_{\omega}$, coefficients $\hat{\boldsymbol{\beta}}_{\omega}$ and predicted response $\hat{\boldsymbol{y}}_{\omega}$.

- Calculate weights for scores and response.

- Center $\operatorname{diag}\left(1 / \omega_{1}, \ldots, 1 / \omega_{n}\right) \boldsymbol{T}_{\omega}$ by the median and scale the columns with the Rousseeuw and Croux [15] robust scale estimator $Q n$ to obtain $\tilde{\boldsymbol{T}}$.

- Calculate distances for $\tilde{\boldsymbol{t}}_{i}(i$ th row of $\tilde{\boldsymbol{T}}$ ) and the robustly centered and scaled residuals $r_{i}$ for $i \in\{1, \ldots, n\}$ :

$$
\begin{aligned}
d_{i} & =\frac{\left\|\tilde{\boldsymbol{t}}_{i}\right\|}{\operatorname{med}_{j}\left\|\tilde{\boldsymbol{t}}_{j}\right\|} \\
r_{i} & =\frac{\left|y_{\omega, i}-\hat{y}_{\omega, i}-\operatorname{med}_{k}\left(y_{\omega, k}-\hat{y}_{\omega, k}\right)\right|}{c \operatorname{med}_{j}\left|y_{\omega, j}-\hat{y}_{\omega, j}-\operatorname{med}_{k}\left(y_{\omega, k}-\hat{y}_{\omega, k}\right)\right|}
\end{aligned}
$$

- Update weights $\omega_{i}=\sqrt{\omega_{T}\left(d_{i}\right) \omega_{R}\left(r_{i}\right)}$.

Repeat until convergence of $\hat{\boldsymbol{\beta}}_{\omega}$.

3. Denote estimates of the final iteration by $\boldsymbol{A}$ and $\hat{\boldsymbol{\beta}}$ and the scores by $\boldsymbol{T}=\boldsymbol{X} \boldsymbol{A}$.

Algorithm 1: ThesSPRM algorithm. 
The algorithm to compute the SPRM estimators iteratively reweights a sparse PLS estimate. This sparse PLS estimate is computed as in Lee et al. [16], who outline a sparse adaptation of the NIPALS computation scheme [17], where in each step of the NIPALS the obtained direction vector of the deflated $\boldsymbol{X}$ matrix is modified according to Equation (14) in order to get sparseness. The starting values of the SPRM algorithm have to be robust. Failing to estimate robust starting values, would lead to an overall nonrobust estimator. Algorithm 1 presents the computing scheme and details the starting values. We iterate until convergence, that is whenever the relative difference in norm between two consecutive approximations of $\hat{\boldsymbol{\beta}}$ is smaller than a specified threshold, e.g. $10^{-2}$. An implementation of the algorithm is available on CRAN in the package sprm [18].

\section{Model selection}

The computation of the SPRM estimator requires specification of $h_{\max }$, the number of latent components, and the sparsity parameter $\eta \in[0,1$ ) (see Equation (14)). For $\eta=0$ the model is estimated including all variables, for $\eta$ tending towards 1 almost no variables are selected.

A grid of values for $\eta$ is searched and $h_{\max }=1,2, \ldots, H$. With $k$-fold robust cross validation the best parameter combination is selected. For each combination of $h_{\max }$ and $\eta$ the model is estimated $k$ times based on a training set containing $(100-k)$ percent of the data, and then evaluated for the remaining data, constituting the validation set. All observations are considered once for validation and so we obtain a single prediction for each of them. As robust cross validation criterion the one sided $\alpha \%$ trimmed mean is calculated from the squared prediction errors, such that the largest $\alpha \%$ errors which may come from outliers, are excluded. We choose the parameter combination where this measure of prediction accuracy is smallest.

The model selection procedure in the following is based on 10-fold cross validation. For the robust methods, the one sided $15 \%$ trimmed mean squared error is applied as decision criterion and for the classical methods the mean squared error of prediction is used for validation. The parameter $h_{\max }$ has a value domain from 1 to 5 and for SPLS and SPRM the sparsity parameter $\eta$ is chosen among ten equally spaced values from 0 to 0.9 . 


\section{Simulation study}

In this section the properties of SPRM and the related methods PRM, PLS and SPLS are studied by means of a simulation study. The predictand is generated according to the model

$$
y_{i}=\boldsymbol{t}_{i} \boldsymbol{\gamma}+e_{i} \quad \text { for } \quad 1 \leq i \leq n,
$$

where the score matrix $\boldsymbol{T}=\boldsymbol{X} \boldsymbol{A}$, for a given matrix of direction vectors $\boldsymbol{A}$.

Let $\boldsymbol{X}$ be an $n \times p$ data matrix with columns generated independently from the standard normal distribution. We generate the columns $\boldsymbol{a}_{h}(h=$ $\left.1, \ldots, h_{\max }\right)$ of $\boldsymbol{A}$ such that only the first $q \leq p$ elements of each $\boldsymbol{a}_{h}$ are nonzero. Thereby, the data matrix $\boldsymbol{X}$ is divided into $q$ columns of relevant variables and $p-q$ columns of uninformative variables. The nonzero part of $\boldsymbol{A}$ is given by the eigenvectors of the matrix $\boldsymbol{X}_{q}^{T} \boldsymbol{X}_{q}$, where $\boldsymbol{X}_{q}$ contains the first $q$ columns of $\boldsymbol{X}$. This ensures that the side conditions for $\boldsymbol{a}_{h}$ hold (see $(11 b)$ ). The components of the regression vector $\gamma \in \mathbb{R}^{h_{\max }}$ are drawn from the uniform distribution on the interval $[0.5,1.5]$. The errors $e_{i}$ are generated as independent values from the standard normal distribution. In a second experiment, we investigate the influence of outliers. The first $10 \%$ of the errors are generated from $N(15,1)$ instead of $N(0,1)$. To induce bad leverage points, the first $5 \%$ of the observations $\boldsymbol{x}_{i}$ are replaced by vectors of random values from $N(5,0.1)$. This will demonstrate the stability of the robust methods when compared to the classical approaches.

In the simulation study, $m_{r e p}=200$ data sets with $n=60$ observations are generated according to (16) for various values of $p$. While $q=6$ is fixed, we will increase $p$ gradually and therefore decrease the signal to noise ratio. This illustrates the effect of uninformative variables on the four model estimation methods and incorporates low dimensional as well as high dimensional settings. For every generated data set we compute the estimator $\hat{\boldsymbol{\beta}}^{j}$ (for $1 \leq j \leq m_{\text {rep }}$ ) with sparsity parameter $\eta$ and $h_{\max }$ selected as described in Section 4. Note that the true coefficients $\boldsymbol{\beta}^{j}$ are different for every simulation run, since every data set is generated with a different regression vector $\gamma$.

Performance Measures: To evaluate the simulation results, the mean squared error (MSE) is used as a measure of the accuracy of the model estimation.

$$
\operatorname{MSE}(\hat{\boldsymbol{\beta}})=\frac{1}{m_{r e p}} \sum_{1 \leq j \leq m_{r e p}}\left\|\hat{\boldsymbol{\beta}}^{j}-\boldsymbol{\beta}^{j}\right\|^{2}
$$


Table 1: Mean percentage of correct zero coefficients, i.e. zero coefficients of uninformative variables, for SPLS and SPRM for simulations with (a) clean training data and (b) training data with $10 \%$ outliers.

\begin{tabular}{llcccc}
\hline \hline$p-q$ & 20 & 100 & 200 & 300 & 500 \\
\hline SPLS & 91.2 & 97.6 & 98.4 & 99.1 & 98.0 \\
SPRM & 75.5 & 93.4 & 95.1 & 96.8 & 94.5 \\
\hline
\end{tabular}

(a) without outliers

\begin{tabular}{llcccc}
\hline \hline$p-q$ & 20 & 100 & 200 & 300 & 500 \\
\hline SPLS & 43.5 & 38.7 & 36.2 & 39.3 & 35.5 \\
SPRM & 76.9 & 91.0 & 94.2 & 98.1 & 97.6 \\
\hline
\end{tabular}

(b) with outliers

Furthermore, let $\hat{\boldsymbol{\beta}}_{0}^{j}$ be the subvector of $\boldsymbol{\beta}^{j}$ corresponding to the uninformative variables. In the true model $\boldsymbol{\beta}_{0}^{j}$ is a vector of zeros. Nonzero values of $\hat{\boldsymbol{\beta}}_{0}^{j}$ contribute to the model uncertainty. One main advantage of sparse estimation is to reduce this uncertainty by setting most coefficients of uninformative variables exactly to zero. The mean number of nonzero values in $\hat{\boldsymbol{\beta}}_{0}^{j}$ is reported for both sparse methods to illustrate whether this goal was achieved. Furthermore, the mean number of nonzero coefficients of the informative variables, is reported.

The last quality criterion discussed in this section is the prediction performance of the estimated model for new data of the same structure. A test data set with $n=60$ observations is generated according to the model in each repetition. For $1 \leq j \leq m_{r e p}$ the estimated response of the test data is denoted by $\hat{\boldsymbol{y}}_{\text {test }}^{j}$ and the true response is $\boldsymbol{y}_{\text {test }}^{j}$. Then the mean squared prediction error (MSPE) is computed as

$$
\mathrm{MSPE}=\frac{1}{m_{r e p}} \sum_{1 \leq j \leq m_{r e p}}\left\|\hat{\boldsymbol{y}}_{\text {test }}^{j}-\boldsymbol{y}_{\text {test }}^{j}\right\|^{2} .
$$

Results for clean data: In the absence of outliers (see Figure 2a and 3a), the overall performance of the classical methods SPLS and PLS is slightly better than for the robust counterparts SPRM and PRM, respectively. In Figure 2a it is seen that the MSE is smallest for SPLS. If all variables are informative, so $p-q=0$, then PLS performs as good as SPLS; but for 
Table 2: Mean percentage of correct nonzero coefficients, i.e. nonzero coefficients of the six informative variables, for SPLS and SPRM for simulations with (a) clean training data and (b) training data with $10 \%$ outliers.

\begin{tabular}{llcccc}
\hline \hline$p-q$ & 20 & 100 & 200 & 300 & 500 \\
\hline SPLS & 65.8 & 54.1 & 52.0 & 48.4 & 46.6 \\
SPRM & 70.0 & 53.8 & 47.8 & 46.7 & 44.8 \\
\hline
\end{tabular}

(a) without outliers

\begin{tabular}{llcccc}
\hline \hline$p-q$ & 20 & 100 & 200 & 300 & 500 \\
\hline SPLS & 65.2 & 70.1 & 71.3 & 68.4 & 72.2 \\
SPRM & 68.8 & 53.8 & 49.3 & 45.0 & 41.3 \\
\hline
\end{tabular}

(b) with outliers

an increasing number of uninformative variables PLS quickly becomes less reliable. The same can be observed for the mean squared prediction error in Figure 3a. Both Figures 2a and 3a show that SPRM is not as accurate as SPLS, but performs much better than PLS and PRM for settings with increasing number of uninformative variables.

Table 1a underpins the advantage of sparse methods. It shows that the average percentage of uninformative variables excluded from the model is close to $100 \%$. SPLS is again slightly better than SPRM, but for both estimates few uninformative variables are included, leading to reduced estimation error in comparison to PLS and PRM. The MSE for the estimation of $\boldsymbol{\beta}_{0}$ is given in Figure 4a. SPLS and SPRM have a comparably good performance, even though SPRM has less zero components in $\hat{\boldsymbol{\beta}}_{0}^{j}$. That means that the nonzero coefficient estimates of the uninformative variables are very small for SPRM. PRM gives surprisingly good results for the MSE of $\hat{\boldsymbol{\beta}}_{0}$ and outperforms PLS. Table 2a shows the mean percentage of nonzero coefficients for the informative variables. For both SPLS and SPRM only roughly half of the six informative variables are included. The sensitivity of SPLS, i.e. the proportion of nonzero correctly identified as such, is reported to be close to $100 \%$ in other simulation settings [11], but in this simulation setting the true nonzero coefficients can be close to zero. SPRM includes slightly less variables, but gives very comparable results to SPLS.

Results for data with outliers: Outliers distort the estimation of PLS and 


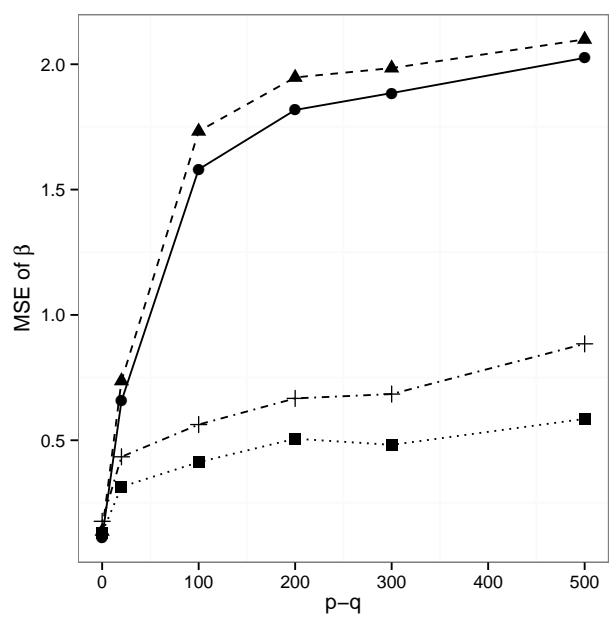

(a) without outliers

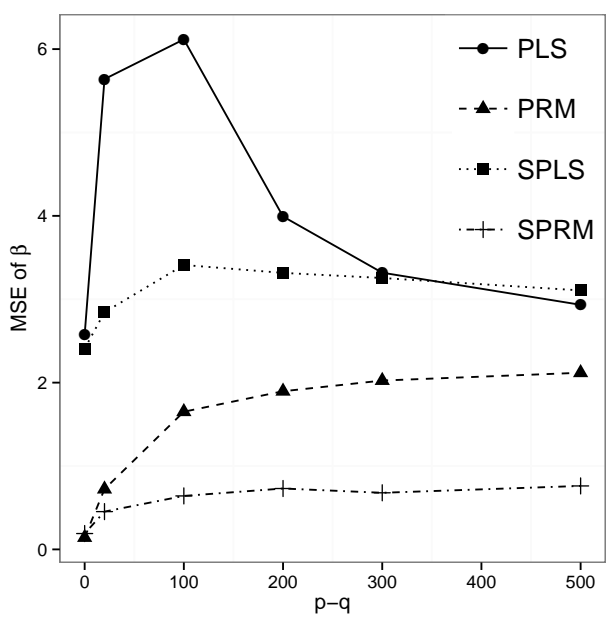

(b) with outliers

Figure 2: Mean squared error of the coefficient estimates for PLS, PRM, SPLS and SPRM for simulations with (a) clean training data and (b) training data with $10 \%$ outliers.

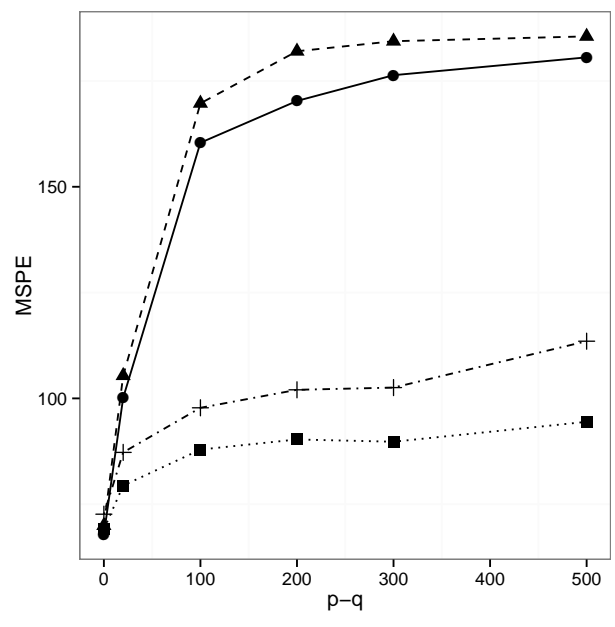

(a) without outliers

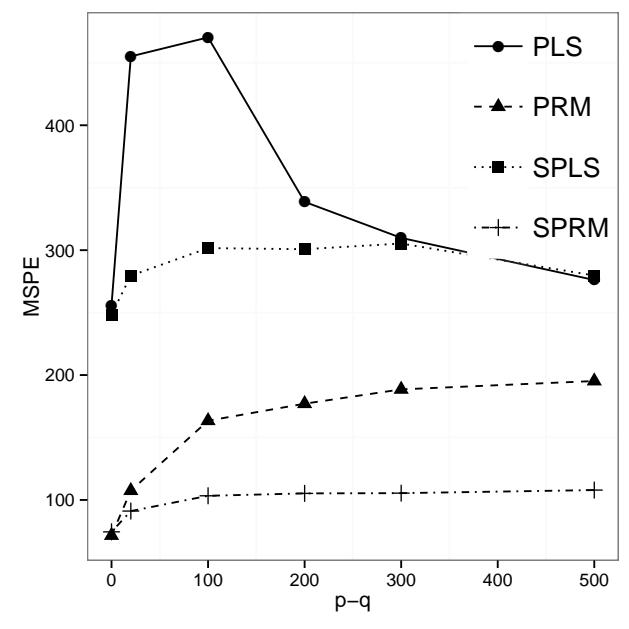

(b) with outliers

Figure 3: Mean squared prediction error for PLS, PRM, SPLS and SPRM for simulations with (a) clean training data and (b) training data with $10 \%$ outliers. 


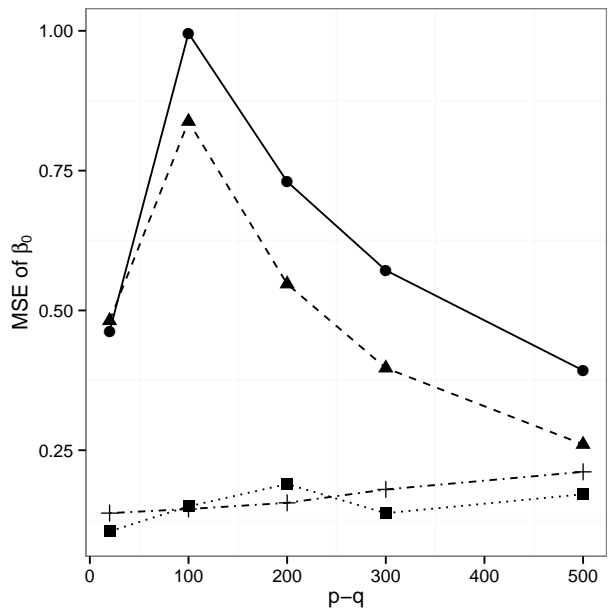

(a) without outliers

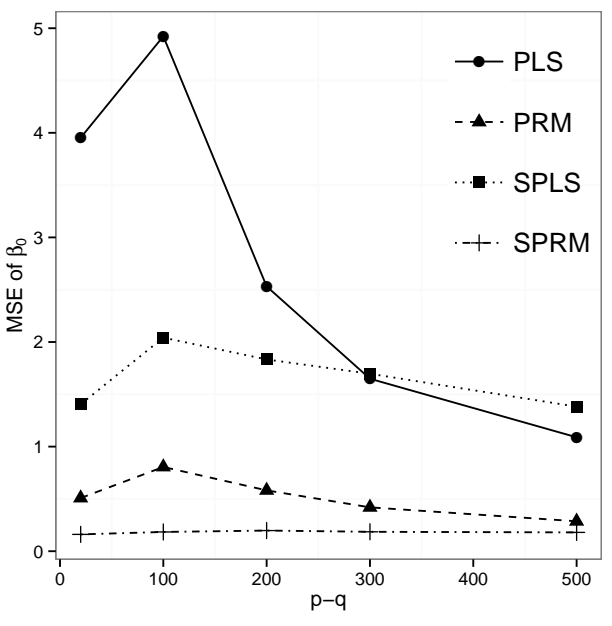

(b) with outliers

Figure 4: Mean squared error of the coefficient estimates of the uninformative variables for PLS, PRM, SPLS and SPRM for simulations with (a) clean training data and (b) training data with $10 \%$ outliers.

SPLS heavily. Figures $2 \mathrm{~b}$ and $3 \mathrm{~b}$ show that the performance of PLS and SPLS strongly deteriorates, while the robust methods are hardly influenced by the presence of the outliers. Furthermore, the robust methods behave as expected as the number of uninformative variables increases: The MSE and MSPE for PRM increase remarkably, whereas SPRM shows only a slight increase, which illustrates the advantage of sparse estimation.

In Table $1 b$ it is seen that SPRM excludes nearly all uninformative variables from the model, whereas SPLS fails to identify them up to a high degree. For all settings, less than half of the uninformative variables are excluded. Hence, the estimation of $\boldsymbol{\beta}_{0}$ is distorted for the classical methods as shown in Figure 4b. Not only the uninformative variables are affected by this trend. In Table $2 \mathrm{~b}$, the average percentage of nonzero coefficients corresponding to informative variables, are shown. From these results, it is evident that SPLS includes more informative variables compared to the case without outliers, which can be explained by the relatively large number of contributing variables in the models. For SPRM only marginal changes are observed compared to the results for data without outliers.

Increasing the number of outliers: An important focus in the analysis of robust methods, is to study how an increasing percentage of outliers affects 


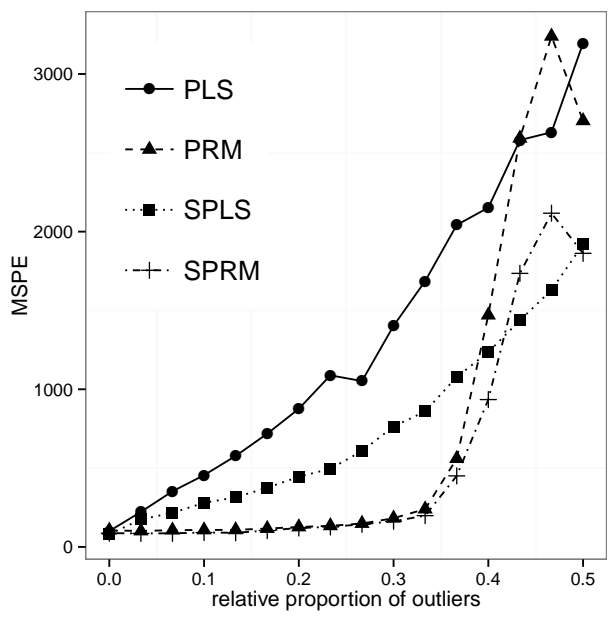

(a) with $p-q=20$

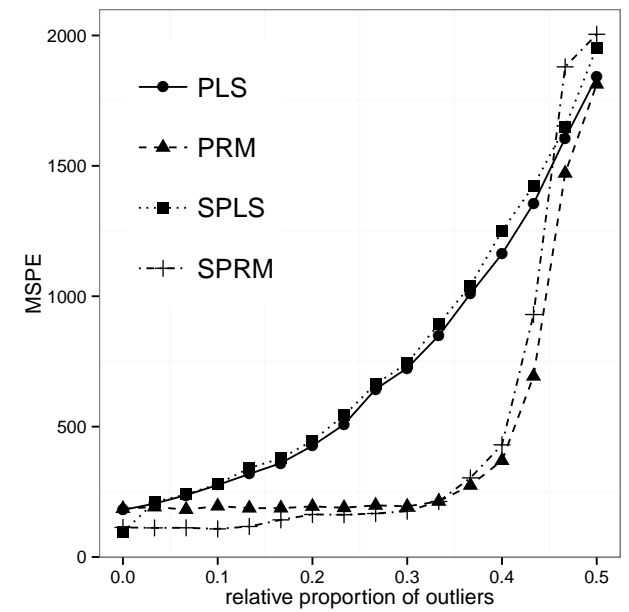

(b) with $p-q=500$

Figure 5: Mean squared prediction error for PLS, PRM, SPLS and SPRM illustrating the effect of increasing number of outliers for data with (a) 20 uninformative variables, (b) 500 uninformative variables.

the model estimation. We use the same simulation design, again with $m_{r e p}=$ 200 repetitions for each considered number of outliers. In each step the number of outliers increases by two (one of these two is a bad leverage point) till $50 \%$ outliers are generated. The mean squared prediction error as defined in (18) is calculated. Figures 5a and 5b display the MSPE for increasing number of outliers, each graph for a fixed number of uninformative variables.

We observe for the robust methods PRM and SPRM hardly any change in the quality of the prediction performance of the estimated models for up to $33 \%$ contamination. The classical methods yield distorted results even for only $3 \%$ contamination. Figure $5 \mathrm{~b}$ show that this high robustness of PRM and SPRM remains when there is a large number of (uninformative) variables. We conclude that the robust methods clearly outperform PLS and SPLS in presence of outliers, while SPRM gives better mean squared prediction error than PRM for percentages of outliers up to 33 percent.

Nonnormal error distributions: A common assumption in model (16) is that the errors $e_{i}$ come from a normal distribution. We simulate data with $p=500, q=6$ and $n=60$ as described previously for the setting without outliers, but replace the error term $e_{i}$ by random values from heavy tailed distributions. In Table 3, the MSE of the coefficients $\operatorname{MSE}(\hat{\boldsymbol{\beta}})$ estimated for 
Table 3: Mean value (and standard error) of the mean squared error of the coefficient estimates $\operatorname{MSE}(\hat{\boldsymbol{\beta}})$ for simulated data with error terms from the standard normal distribution, the $t$ distribution with 3 and 2 degrees of freedom and Laplace distribution with dispersion parameter 1 and 2 .

\begin{tabular}{|c|c|c|c|c|c|c|c|c|c|c|}
\hline & \multicolumn{2}{|l|}{$\mathrm{N}$} & $t_{3}$ & \multicolumn{3}{|c|}{$t_{2}$} & L1 & \multicolumn{3}{|c|}{ L2 } \\
\hline PLS & 2.00 & $(.05)$ & 2.25 & $(.06)$ & 2.77 & $(.11)$ & 2.04 & $(.05)$ & 2.72 & $(.05)$ \\
\hline PRM & 2.10 & (.05) & 2.13 & $(.05)$ & 2.18 & $(.05)$ & 2.06 & $(.05)$ & 2.31 & $(.06)$ \\
\hline SPLS & 0.60 & $(.02)$ & 1.29 & $(.07)$ & 2.29 & $(.15)$ & 1.06 & $(.04)$ & 2.94 & (.09) \\
\hline SPRM & 0.88 & $(.04)$ & 1.16 & $(.05)$ & 1.22 & $(.05)$ & 1.15 & $(.05)$ & 2.43 & $(.09)$ \\
\hline
\end{tabular}

simulated data with normal error terms are compared to those from data with error terms from the t distribution with 3 and 2 degrees of freedom and the Laplace distribution with a dispersion parameter of 1 and of 2 . The mean squared error of the coefficient estimates behaves as expected for the $\mathrm{t}$ distributions. It increases significantly for the classical methods as the number of degrees of freedom decreases from three to two, in which case more extreme values are generated. For error terms from the Laplace distribution, the advantage of the robust methods gets more pronounced for the Lapalce distribution with the higher dispersion parameter, which generates more extreme values.

Computation time and convergence: The computation time of the robust sparse NIPALS algorithm strongly depends on the number of iterations needed till convergence of the robust and sparse coefficient estimates $\hat{\boldsymbol{\beta}}_{\omega}$ as described in Algorithm 1 in step 2. This varies with the structure of the data. In the simulation study with ten percent outliers in the data and $p=500$ on average five iterations are needed, i.e. the sparse NIPALS algorithm, which is computationally efficient for a univariate response, has to be applied on average five times. On a standard PC (Intel i7-4790K) the average computation time for the estimation of a model based on these data with fixed parameters $\eta=0.5$ and $h_{\max }=2$ is 0.12 seconds.

\section{Application}

Sparse regression methods and big data go hand in hand. Therefore, there are manifold applications of those methods in the omics fields (e.g. the microarray CHIP-chip data [11]), but they have also found their way into chemometrics [e.g. 19] or medicine (e.g. the application on NMR spectra 


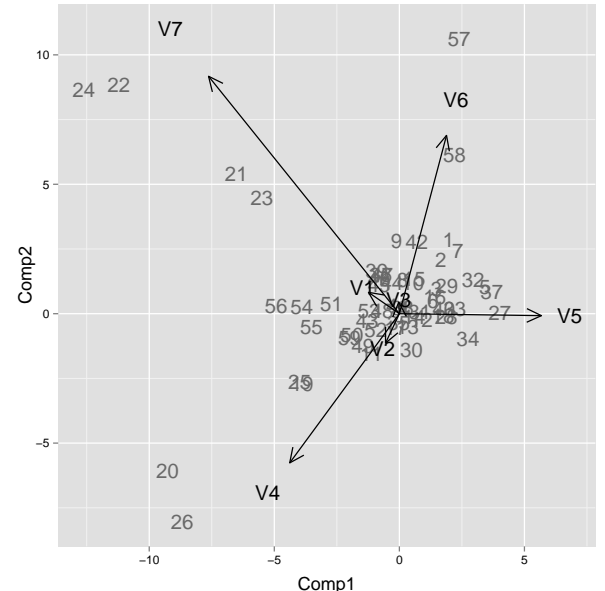

(a) PRM

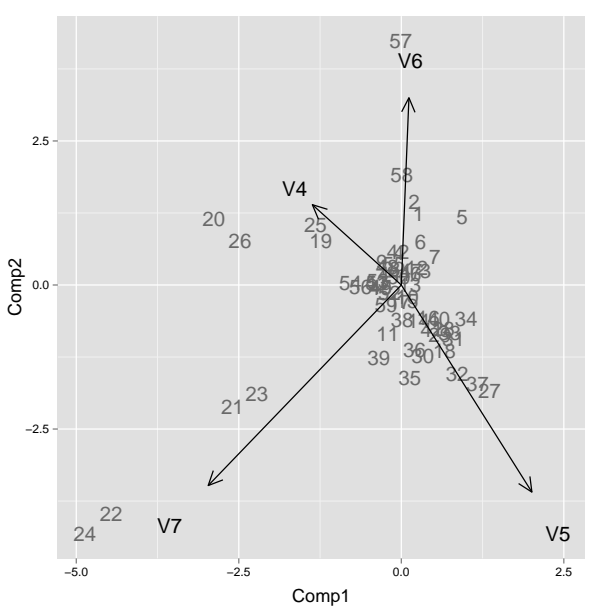

(b) SPRM

Figure 6: The PRM and SPRM biplots for the gloss data example

of neural cells [12]). Even though sparse regression methods are of great use when data dimensionality is high, they can already be beneficial when applied to low dimensional problems (which, in the context of classification, has been reported in Filzmoser et al. [19]). Therefore, in the first example we will focus on data of moderate dimensionality, followed by a gene expression example to illustrate the application to high dimensional data.

The gloss data: The data consist of $n=58$ polymer stabilization formulations, wherein the $p=7$ predictors are the respective concentrations of seven different classes of stabilizers. The actual nature of the classes of stabilizers, as well as the respective concentrations, are proprietary to BASF Corp. and cannot be disclosed. The response variable targets to quantify the quality of stabilization by measuring how long it takes for the polymer to lose $50 \%$ of its gloss when weathered (in what follows, simply called the gloss). The target is to predict the gloss from the stabilizer formulations. The data were scaled with the $Q n$ scale for the robust methods [15] and for the classical methods with the standard deviation.

Table 4: Prediction performance for polymer stabilizer data.

\begin{tabular}{ccccc}
\hline \hline & PLS & PRM & SPLS & SPRM \\
\hline 15\% TMSPE & 2099382 & 2218181 & 2113960 & 2047858 \\
\hline
\end{tabular}


PLS, SPLS, PRM and SPRM use the 10-fold cross validation procedure described in Section 4. The optimal number of latent components for PLS and PRM was detected to equal 1. For SPRM the optimal number of latent components is 4 and the sparsity parameter was found to be $\eta=0.6$; for SPLS we have $h_{\max }=3$ and $\eta=0.9$.

To evaluate the four methods, leave-one-out cross validation was performed and the one sided $15 \%$ trimmed mean squared prediction error (TMSPE) is reported in Table 4. SPRM performs slightly better according to the TMSPE. Another advantage of sparse robust modeling in this example is the interpretability. Figure 6 compares the biplots of PRM and SPRM for the first two latent components. In the sparse biplot variables V1, V2 and V3 are excluded and so it is easier to grasp in which way the latent components depend on the original variables, and how the individual cases differ with respect to the selected variables.

The NCI data: The National Cancer Institute provides data sets of measurements from 60 human cancer cell lines (http://discover.nci.nih. gov/cellminer/). The 40th observation has to be excluded due to missing values, i.e. $n=59$. The gene expression data comes from an Affymetrix HG-U133A chip and was normalized with the GCRMA method. It is used to model $\log _{2}$ transformed protein expression from a Lysate Array. From the gene expression data only the $25 \%$ of the variables with highest variance are considered, which leads to $p=5571$, as was similarly conducted by [16]. The protein data consists of measurements of 162 expression levels. Since the proposed method is designed for univariate response we modeled the relationship for each protein expression separately and obtain 162 models for each of the competitive methods.

As before, the model selection is done using 10-fold cross validation (see Section 4) and the selected models are evaluated with the 15\% TMSPE. For each of the 162 different responses the TMSPE of each estimated model is divided by the smallest of the four TMSPEs. This normed TMSPE is a value equal to 1 (for the best method) or larger and we can compare it across the different responses (see Figure 7). Overall, the combination of sparsity and robustness leads to a superior evaluation. The median of the normed TMSPE of the SPRM models is very close to 1 and therefore, we can conclude that for half of the models SPRM is either the best or very close to the best model. PLS is not an appropriate method for these data, since the TMSPE differs strongly from the best model in most cases. 


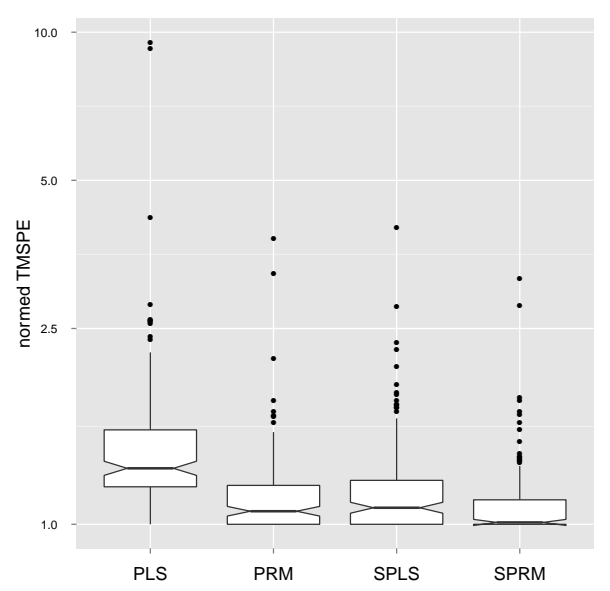

Figure 7: Boxplots of normed TMSPE of 162 responses from the NCI data for PLS, PRM, SPLS and SPRM.

For purpose of illustration, we focus on Keratin 18 as response. It has the highest variance of all responses and its expression is an often used criterion for the detection of carcinomas [20]. Table 5 presents the number of latent components and the number of selected variables (i.e. having nonzero estimated coefficients) for each method, together with the TMSPE. The SPRM model gives the best result with only 6 out of 5571 variables selected. Even PRM performs better than SPLS in this high dimensional setting, which underpins the importance of robust estimation for these data. Figure 8 shows the biplot of scores and directions for the first two latent components of the SPLS and the SPRM model. For SPRM, the first latent component is determined by the variables KRT8 and KRT19. The expression of these genes is known to be closely related to the protein expression of Keratin 18 and they are used for the identification and classification of tumor cells [21, 20]. KRT8 has previously been reported to play an important role in sparse and robust regression models of these data [4]. The biplot further unveils some clustering in the scores and provides insight into the multivariate structure of the data. The biplot of the SPLS model (Figure 8a) cannot be interpreted since this model including 78 variables is too complex. Interestingly, in the SPLS biplot KRT8 and KRT19 are also the genes which have the largest positive influence on the first latent component.

Note that the case weights $\omega_{i}$ of the robust models presented in Figure 9 are as expected: they are one for the bulk of the data, exactly zero for the 
Table 5: Model properties for NCI gene expression data with protein expression of Keratin 18 as response variable.

\begin{tabular}{lcccc}
\hline \hline & PLS & PRM & SPLS & SPRM \\
\hline 15\% TMSPE & 3.22 & 1.72 & 2.03 & 1.24 \\
no. of latent components & 4 & 2 & 2 & 3 \\
no. of selected variables & 5571 & 5571 & 78 & 6 \\
\hline
\end{tabular}

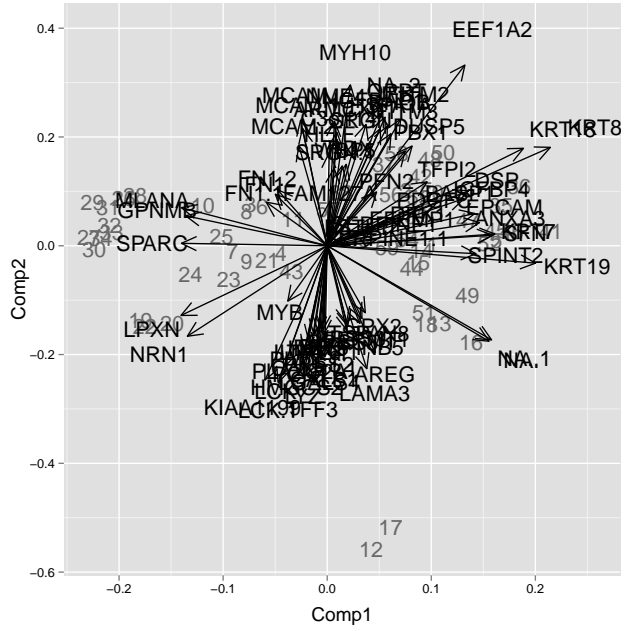

(a) SPLS

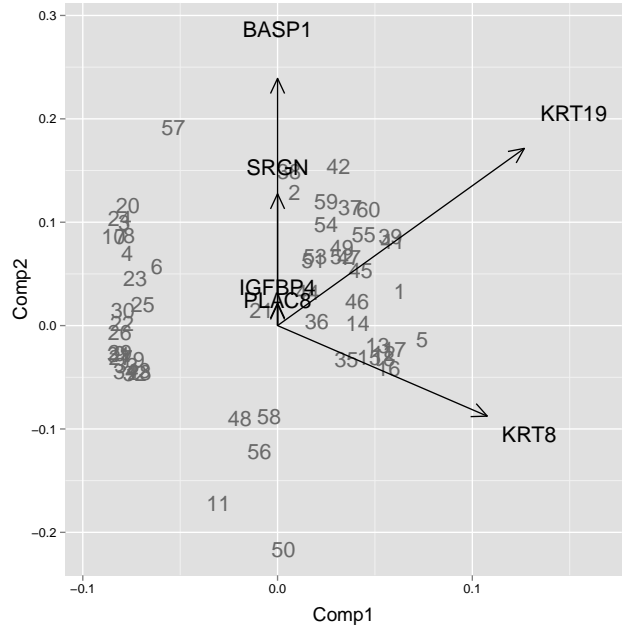

(b) SPRM

Figure 8: The SPLS and SPRM biplots for the gene data example with protein expression of Keratin 18 as response.

potential outliers and in the interval $(0,1)$ for a few observations, which is an immediate consequence of adopting the Hampel weighting function (Equation (15) and Figure 1). Hence, outliers can easily be identified. The detection of potential outliers differs between PRM and SPRM, but the pattern is similar.

\section{Conclusions}

SPRM is a sparse and robust regression method, which performs dimension reduction in a manner closely related to partial least squares regression. It performs intrinsic variable selection and retrieves sparse latent components, which can be visualized in biplots and interpreted better than nonsparse latent components especially for high dimensional data. Since sparse 


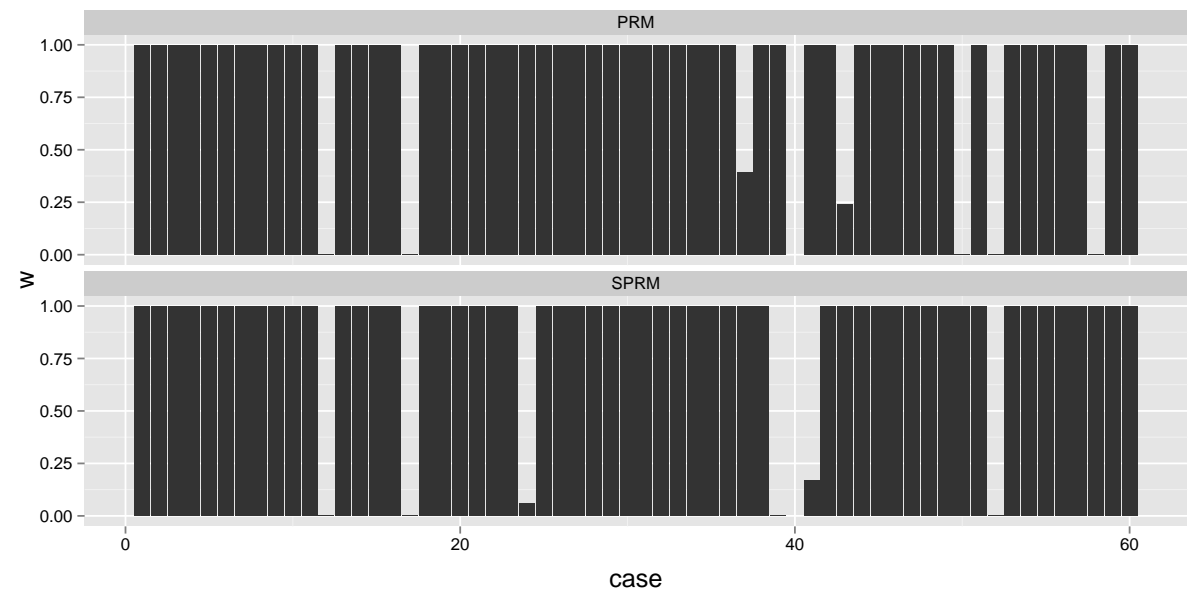

Figure 9: The PRM and SPRM case weights for the gene data example with protein expression of Keratin 18 as response.

methods eliminate the uninformative variables, higher estimation and prediction accuracy is attained. The SPRM estimation of latent components and the selection of variables is resistant to outliers. To reduce the influence of outliers on the model estimation, an iteratively reweighted regression algorithm is used. The resulting case weights can be used for outlier diagnostics.

We demonstrated the importance of robustness and sparsity properties in a simulation study. The method was shown to be robust with respect to outliers in the predictors and in the response and achieved good results for settings with high percentage of outliers. The informative variables were detected accurately. We illustrated the performance of SPRM on a data set of polymer stabilization formulations of moderate dimensionality and on high dimensional gene expression data. An implementation of the SRPM, as well as visualization tools and the cross-validation model selection method outlined in Section 4, is available on CRAN in the package sprm [18].

The extension of SPRM regression for a multivariate response is a next step to take. Note that few papers combine sparseness and robustness for multivariate statistics, an exception is Croux et al. [22] for principal component analysis. The development of prediction intervals around the SPRM prediction is another challenge left for future research. A bootstrap approach seems reasonable, but its validity remains to be investigated. Obtaining theoretical results on breakdown point or consistency of the model section is out of the scope of this paper. Few theoretical results are available in the PLS lit- 
erature, and this only for the nonrobust and nonsparse case. In this paper we proposed and put into practice a new sparse and robust partial least squares method, which we believe to be valuable for data scientists confronted with prediction problems involving many predictors and noisy data.

\section{Acknowledgments}

This work is supported by BASF Corporation and the Austrain Science Fund (FWF), project P 26871-N20.

\section{References}

[1] R. Tibshirani. Regression shrinkage and selection via the lasso. Journal of the Royal Statistical Society, B, 58:267-288, 1996.

[2] R. Tibshirani. Regression shrinkage and selection via the lasso: a retrospective. Journal of the Royal Statistical Society, B, 73(3):273-282, 2011.

[3] R. Maronna, D. Martin, and V. Yohai. Robust Statistics. John Wiley \& Sons, 2006.

[4] A. Alfons, C. Croux, and S. Gelper. Sparse least trimmed squares regression for analyzing high-dimensional large data sets. The Annals of Applied Statistics, 7:226-248, 2013.

[5] P. J. Rousseeuw and A. M. Leroy. Robust Regression and Outlier Detection. John Wiley \& Sons, 2nd edition, 2003.

[6] H. Wold. Multivariate analysis. In P.R. Krishnaiah, editor, Proceedings of an International Symposium 14-19 June, pages 391-420. Academic Press, NY, 1965.

[7] P. Filzmoser, S. Serneels, R. Maronna, and P.J. Van Espen. Robust multivariate methods in chemometrics. In S.D. Brown, R. Tauler, and B. Walczak, editors, Comprehensive Chemometrics, volume 3, pages 681-722. Elsevier, Oxford, 2009.

[8] S. Serneels, C. Croux, P. Filzmoser, and P.J. Van Espen. Partial robust M-regression. Chemometrics and Intelligent Laboratory Systems, 79:5564, 2005. 
[9] B. Liebmann, P. Filzmoser, and K. Varmuza. Robust and classical pls regression compared. Journal of Chemometrics, 24:111-120, 2010.

[10] K. Lê Cao, D. Rossouw, C. Robert-Granié, and P. Besse. A sparse pls for variable selection when integrating omics data. Statistical Applications in Genetics and Molecular Biology, 7:35, 2008.

[11] H. Chun and S. Keleş. Sparse partial least squares regression for simultaneous dimension reduction and variable selection. Journal of the Royal Statistical Society, B, 72:3-25, 2010.

[12] G.I. Allen, C. Peterson, M. Vannucci, and M. Maletić-Savatić. Regularized partial least squares with an application to nmr spectroscopy. Statistical Analysis and Data Mining, 6:302-314, 2013.

[13] H. Zou, T. Hastie, and R. Tibshirani. Sparse principal component analysis. Journal of Computational and Graphical Statistics, 15:265-286, 2006.

[14] F.R. Hampel, E.M. Ronchetti, P.J. Rousseeuw, and W.A. Stahel. Robust Statistics: The Approach Based on Influence Functions. Wiley, 1986.

[15] P.J. Rousseeuw and C. Croux. Alternatives to the median absolute deviation. Journal of the American Statistical Association, 88:1273$1283,1993$.

[16] Donghwan Lee, Woojoo Lee, Youngjo Lee, and Yudi Pawitan. Sparse partial least-squares regression and its applications to high-throughput data analysis. Chemometrics and Intelligent Laboratory Systems, 109 (1):1-8, 2011.

[17] H. Wold. Soft Modeling by Latent Variables; the Nonlinear Iterative Partial Least Squares Approach. Perspectives in Probability and Statistics. Papers in Honour of M. S. Bartlett, 1975.

[18] S. Serneels and I. Hoffmann. sprm: Sparse and Non-sparse Partial Robust $M$ Regression, 2014. R package version 1.0.

[19] P. Filzmoser, M. Gschwandtner, and V. Todorov. Review of sparse methods in regression and classification with application in chemometrics. Journal of Chemometrics, 26:42-51, 2012. 
[20] R. G. Oshima, H. Baribault, and C. Caulín. Oncogenic regulation and function of keratins 8 and 18. Cancer and Metastasis Reviews, 15(4): 445-471, 1996.

[21] L. J. Schelfhout, G. N. Van Muijen, and G. J. Fleuren. Expression of keratin 19 distinguishes papillary thyroid carcinoma from follicular carcinomas and follicular thyroid adenoma. American Journal of Clinical Pathology, 92(5):654-658, 1989.

[22] Christophe Croux, Peter Filzmoser, and Heinrich Fritz. Robust sparse principal component analysis. Technometrics, 55(2):202-214, 2013. 